Review

\title{
Depicting the Landscape of Adipose Tissue-Specific Macrophages and Their Immunometabolic Signatures during Obesity
}

\author{
Hannelie Korf 1,*, Markus Boesch ${ }^{1}$, Rita Feio-Azevedo ${ }^{1}$, Lena Smets ${ }^{1}$, \\ Roselien Vandecasteele ${ }^{1}$, Schalk van der Merwe ${ }^{1,2, *}$ \\ 1 Laboratory of Hepatology, CHROMETA Department, KU Leuven, Leuven 3000, \\ Belgium \\ 2 Department of Gastroenterology and Hepatology, UZ Leuven, Leuven 3000, \\ Belgium \\ * Correspondence: Hannelie Korf, Email: hannelie.korf@kuleuven.be; \\ Schalk van der Merwe, Email: schalk.vandermerwe@uzleuven.be.
}

\begin{abstract}
Obesity is a widespread health condition, which can lead to the development of metabolic disorders, such as type 2 diabetes mellitus, nonalcoholic fatty liver disease and cardiovascular diseases. Obesity is marked by the excessive deposition of fat in adipose tissue sites combined with chronic low-grade inflammation. Within this clinical setting, it is well established that adipose tissue macrophages exhibit prominent roles in regulating inflammation and metabolism. However, aside from these wellestablished roles, the involvement of microenvironmental cues as well as underlying cellular metabolism in driving immunological fate decisions within macrophages are poorly understood. Here we aim to map the different adipose tissue-derived macrophage subsets, together with their metabolic and functional profiles. Finally, we discuss their potential contribution during homeostasis and disease progression associated with obesity.
\end{abstract}

KEYWORDS: adipose tissue macrophages; obesity; immunometabolism; microenvironmental niche

\section{G Open Access}

Received: 05 August 2019

Accepted: 25 November 2019

Published: 26 November 2019

Copyright (c) 2019 by the author(s). Licensee Hapres, London, United Kingdom. This is an open access article distributed under the terms and conditions of Creative Commons Attribution 4.0 International License.

\section{INTRODUCTION}

Obesity is a global health pandemic that predisposes individuals to develop metabolic disorders, such as type 2 diabetes mellitus and nonalcoholic fatty liver disease (NAFLD) [1]. In order to face this escalating disease burden, there is an urgent need to unravel the molecular mechanisms that prevent the life-threatening metabolic comorbidities in this expanding patient population and to define novel targets for early therapeutic intervention. Although the disease mechanisms remain incompletely understood, it is well established that early inflammatory events within the expanding adipose tissue compartment significantly contribute to dysregulation of metabolic homeostasis [2]. For example, 
adipose tissue can secrete a variety of adipokines that influence inflammation and insulin resistance. Macrophages and their proinflammatory cytokine repertoire are prominent contributors to the adipose tissue secretome and have been identified as key early driver of insulin resistance [3-6]. Furthermore, adipose tissue macrophages can directly influence recruitment of immune cells from the circulation into adipose tissue sites but also in distal organs such as the liver [7-10], and their frequencies correlate with hepatic histopathological severity [11]. Consequently, disruption of macrophages, or their functions, improves insulin sensitivity and abrogated hepatic inflammation and steatosis [12-14]. These findings support a role for macrophages and adipose tissue inflammation in metabolic disorders such as obesity and its complications.

Macrophages are highly diverse cells in terms of functionality, although they all share a common core program directed by lineagespecific transcription factors [15]. Macrophages can be either embryonically seeded in organs where they are maintained through selfrenewal [16], or derived from infiltrating bone marrow monocyte precursors [17,18]. Regardless of their origin, they adapt to microenvironmental cues within the niche they reside in and become imprinted with a unique transcriptional signature [19,20]. Technologies such as single-cell RNA sequencing accelerated discoveries in the field and even implicated a variety of niche-specific macrophages co-existing within one organ [21]. Importantly, these macrophage subsets are specialized in exerting functions such as phagocytosis of apoptotic/necrotic cells, secretion of cytokines and growth factors and remodeling of the extracellular matrix, and all of these processes require mobilization of specific intrinsic metabolic processes [22]. Metabolic repurposing could therefore potentially aid in fine-tuning and correcting macrophage malfunctions during a diseased condition. Notably, as proof of concept, our group recently demonstrated that metabolic rewiring improved monocyte functionality in patients with acute-on-chronic liver failure [23]. Nevertheless, it remains poorly understood how these microenvironmental cues affect immune-metabolic functions of macrophages, especially within the lipid overload setting of obesity and its complications. Here we provide an overview of different subtissular niche macrophages with a focus on their immune-metabolic profile within the adipose depot and discuss their potential contribution during disease progression during obesity.

\section{REVISITING THE PREVIOUS CONCEPT OF MACROPHAGE POLARIZATION IN OBESE ADIPOSE TISSUE}

More than a decade ago, some studies suggested that healthy adipose tissue contains alternatively activated macrophages (M2-like). M2-like macrophages exhibit an anti-inflammatory function through the actions of Interleukin-10 (IL-10) and signal transducer and activator of transcription 3 (STAT3) [24]. Additionally, they maintain insulin sensitivity 
through peroxisome proliferator-activated receptor gamma (PPAR $\gamma$ ), which promotes tissue remodeling and consequently resolves inflammation [25,26]. In support of this concept, interfering with M2-like activation by inhibiting expression of parameters within the downstream IL-4 receptor signaling pathway hampered insulin sensitivity via components such as PPAR $\gamma$, PPAR $\delta$ and KLF4 [25,27,28]. To support these homeostatic and restorative functions, M2-like macrophages exhibit a metabolic profile that relies on fatty acid oxidation (FAO) to fuel tricarboxylic acid (TCA) cycle-coupled oxidative phosphorylation [29], which relies on free intracellular coenzyme A ( $\mathrm{CoA}$ ) availability as a regulator of oxidation, as recently been described [30,31]. Furthermore, a role for the mammalian target of rapamycin complex 2 (mTORC2) and interferon regulatory factor 4 (IRF4) in M2 polarization have been demonstrated. Towards this end, elevated expression of mTORC2 and IRF4 increased glucose-dependent oxidative phosphorylation and upregulated the characteristic M2 parameters arginase 1 and resistin-like molecule a [32].

In contrast, classically activated (M1-like) macrophages are recruited and retained in adipose tissue in NAFLD subjects, where they secrete proinflammatory mediators and disturb insulin sensitivity [6,33,34]. Deletion of the pro-inflammatory signaling molecule IKK $\beta$ in myeloid cells preserved insulin sensitivity and reduced adipose tissue inflammation [13]. Similarly, macrophage-specific deletion of stress-activated c-Jun NH2 terminal kinases, JNK, protects against high-fat diet-induced obesity and insulin resistance and reverted M1 polarization [35]. To meet the energy demand required to exert these acute inflammatory actions, M1 macrophages rely on increased glucose uptake and glycolytic flux [29]. They also feature an interrupted TCA cycle whereby citrate and succinate intermediates accumulate within the cell [36]. Interestingly, the build-up of succinate leads to stabilization of hypoxia-inducible factor (HIF)-1a, a master transcriptional regulator of pro-inflammatory and glycolytic genes [37]. HIF-1a regulates important downstream target genes such as the glucose transporters, GLUT1 and GLUT3 [38], as well as the pyruvate dehydrogenase kinase 1 (PDK1), a metabolic checkpoint directing glucose metabolism towards glycolysis and away from oxidative phosphorylation [39,40]. Finally, macrophage HIF-1a was increased in human and mouse Nonalcoholic steatohepatitis subjects and deletion of myeloid HIF-1a impaired macrophage pro-inflammatory function and adipose tissue inflammation while restoring glucose tolerance [41-43].

Notably, in the in vivo setting and especially in obesity, many different stressors can lead to the activation of inflammatory pathways within adipose tissue macrophages [44]. In addition, macrophages can reside in very different subtissular microenvironments where they give rise to a spectrum of subtypes not accounted for in the oversimplified M1/M2 paradigm. Thereby, macrophages can be located in hypoxic regions or can be found in the vicinity of blood vessels, -nerve fibers, -extracellular matrix or -dying adipocytes. To this end, several studies have 
demonstrated that macrophages exhibit distinct transcriptional signatures and epigenetic traits that are specific to their location [45-47]. This underscores the role of tissue factors in imprinting the macrophage transcriptional program and consequently underlines the importance of studying these tissue microenvironments during obesity and its complications.

\section{REFINING MACROPHAGE FUNCTIONAL STATES BASED ON THE MICROENVIRONMENTAL CUES THEY ENCOUNTER}

\section{Metabolic Activated Macrophages-Metabolism in Overdrive}

As mentioned above, we are only starting to grasp the extent of these macrophage phenotypes and their accompanying metabolic signatures. These various macrophage phenotypes located in the adipose tissue are depicted in Figure 1 and further reviewed here. For instance, it is possible that lipids released by dying adipocytes are excessively engulfed by resident adipose tissue macrophages giving rise to unique macrophage phenotypes. Indeed, recent studies have characterized metabolic signatures of murine adipose tissue, defining two new phenotypes, termed redox-regulatory (Mox) and metabolically activated (MMe) macrophages. Mox macrophages feature enhanced antioxidant gene expression profiles and a quiescent metabolic state, and were reported to be present primarily in lean adipose tissue following truncated oxidized lipid exposure [48]. On the contrary, obese adipose tissue macrophages featured a metabolically activated state (MMe) with increased lipid catabolism and lysosomal biogenesis [49]. Genes such as perilipin-2 and lysosome-associated membrane protein 2 were specifically upregulated in these macrophages. Notably, a cocktail of glucose, insulin and palmitate could recapitulate this metabolically activated state in vitro [50]. Furthermore, MMe macrophages featured a mixed metabolic program with simultaneous upregulation of glycolysis and oxidative phosphorylation pathways following saturated fatty acid exposure. By promoting inflammatory cytokine production as well as lysosomal exocytosis to adipocytes they also participated in both detrimental and beneficial actions during obesity [51]. Additional studies merging metabolomics, transcriptomics and subtissular locations will be critical in further unravelling the potential heterogeneity within MMe macrophages. 

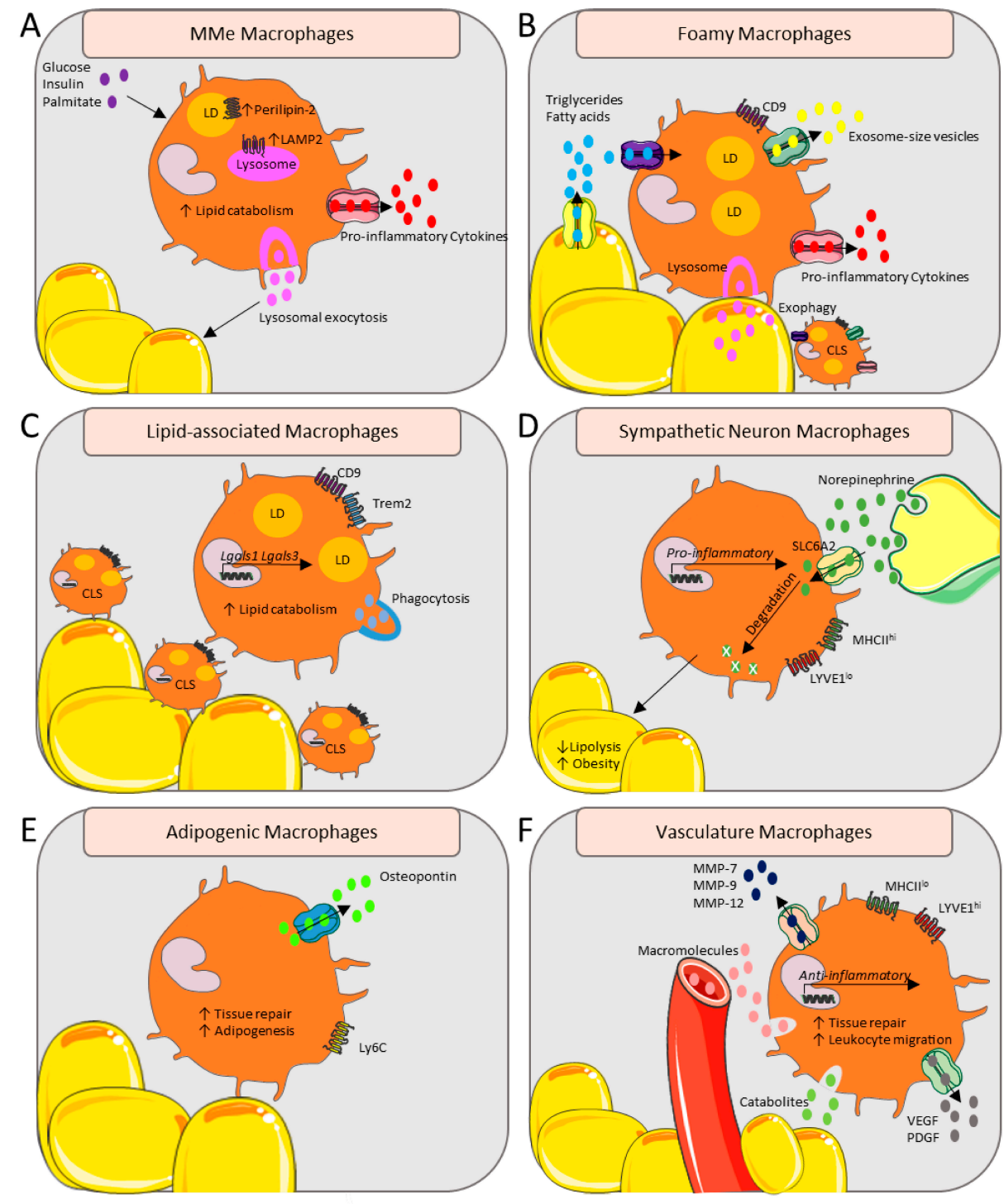

Figure 1. Functional and metabolic overview of adipose tissue macrophages in their subtissular niches. (A) Glucose, insulin and palmitate can induce the metabolically activated (MMe) macrophage state with upregulated Perilipin-2 and LAMP2, leading to increased pro-inflammatory cytokine secretion and inducing lysosomal exocytosis. (B) CD9 ${ }^{+}$Foamy macrophages form a CLS around the adipocytes, and take up triglycerides and fatty acids released by stressed hypertrophic adipocytes resulting in secretion of exosome-size vesicles and pro-inflammatory cytokines. Macrophages release lysosomal content by forming hydrolytic synapses with stressed adipocytes, called exophagy. (C) The CD9 ${ }^{+}$lipid-associated macrophages form a CLS and transcribe Lgals1 and Lgals3. The Trem2 lipid sensor drives phagocytosis and lipid catabolism. (D) Norepinephrine, released by sympathetic neurons, is transported via SLC6A2 and is degraded by the sympathetic neuron macrophages. This leads to an upregulated transcription of proinflammatory genes and increased obesity. (E) Adipogenic macrophages over-express osteopontin and are involved in tissue repair and adipogenesis. (F) The Lyve $1^{\text {hi }}$ vasculature-associated macrophages are localized with the vasculature, engulfing blood-born macromolecules and noxious catabolites, while secreting MMP-7, MMP-9, MMP-12, VEGF and PDGF. Their anti-inflammatory gene transcription profile ensures an involvement in tissue repair and leukocyte migration. CLS, Crown-like structure; LAMP2, Lysosome-associated membrane protein 2; LD, Lipid droplet; LYVE1, Lymphatic vessel endothelial receptor 1; MMe, metabolically activated; MMP-(7, 9, 12), Matrix-metalloproteinase-(7, 9, 12); PDGF, Plateletderived growth factor; SLC6A2, Sodium-dependent noradrenaline transporter; VEGF, Vascular endothelial growth factor. 


\section{Crown-like Structure (CLS) Macrophages-Facing the Heat Zone}

Foamy macrophages-clearing the dead and fueling inflammation and systemic insulin resistance

Under normal conditions, resident adipose tissue macrophages phagocytose adipocyte debris in order to maintain normal adipocyte turnover and tissue homeostasis. However, during obesity progression, adipocytes undergo plasma membrane rupture, endoplasmic reticulum stress and necrosis-like death [33,52]. Following this event, macrophages rapidly surround and engulf the dying adipocytes, thereby creating a characteristic microenvironment known as a crown-like structure (CLS). Because of the size of the stressed hypertrophied adipocytes, macrophages implement an innovative mechanism by forming hydrolytic synapses in which they secrete their lysosomal content to ingest the dying adipocytesa process termed exophagy [53]. The enlarged insulin-resistant adipocytes also release triglycerides and non-esterified fatty acids that are continuously scavenged by surrounding macrophages. Combined, this heightened demand on their endocytic capacity may overwhelm the underlying metabolic state giving rise to metabolically activated macrophages (as described above) [49-51]. Additionally, this process may impede efficient dead cell clearance, similar to what happens in foam cells within atherosclerotic plaques [54], culminating in an aberrant inflammatory response [55]. Important differences between foamy macrophages described here and those present in atherosclerotic plaques also exist. For example, excessive lipid loading appears to suppress inflammatory responses due to a defective pentose phosphate pathway [56-58].

During obesity however, an important study by Hill et al. describes a $\mathrm{CD}^{+}$CLS macrophage population that exhibit a crucial role in storage of excess lipids and that express genes related to lysosomal-dependent lipid metabolism [59]. Interestingly, the authors of this latter study suggest that $\mathrm{CD}^{+}$macrophages metabolically resemble activated macrophages (see above) as well as the previously described CD11 $\mathrm{c}^{\text {high }}$ macrophages [60], since they similarly reside predominantly in the CLS and secrete pro-inflammatory cytokines. Implicating a pathogenic role for these macrophages, the authors demonstrated that adoptive transfer of $\mathrm{CD}^{+}$ macrophages into healthy animals was sufficient to propagate obesityassociated adipose tissue inflammation [59]. $\mathrm{CD}^{+}$macrophages also seem to play a role in regulating metabolism through secretion of exosome-size vesicles. Indeed, miRNA containing extracellular vesicles (e.g., miR-155) constitute an important part of the adipose tissue macrophage secretome and act as a signaling mechanism to regulate local and systemic insulin signaling [61]. 


\section{Lipid-associated macrophages-regulators of adipocyte hypertrophy}

Further refining the diversity of macrophages during obesity, an elegant study by the group of Ido Amit characterized a number of distinct myeloid cell populations within adipose tissue [62]. One population resembled the transcriptional profile of interstitial perivascular macrophages (described below) [63], and another fitted the signature described by Hill et al., described as $\mathrm{CD}^{+}$macrophages (described above) [59]. Strikingly, their results implicate the presence of a third unique macrophage subset, which they termed lipid-associated macrophages (LAMs). The authors demonstrate that LAMs are derived from circulating monocytes and arise specifically under obesity conditions where they are positioned around enlarged adipocytes in crown-like structures [62]. Although also expressing CD9, LAMs seem to be distinct from the pro-inflammatory subset described by Hill et al., and express genes associated with immune suppression such as Lgals1 and Lgals3 $[59,62]$. The transcriptional signature of LAMs is very close to that described for disease-associated microglia (DAM) cells in the brains of subjects with neurodegenerative disorders and in aortic macrophages during atherosclerosis $[64,65]$. Another important finding was that LAMs played a crucial role in preventing adipocyte hypertrophy through a Trem2-mediated mechanism. Trem2 acted as a lipid sensor, driving a gene expression program involved in phagocytosis, lipid catabolism and energy metabolism [62]. Abrogating Trem2 signaling caused massive adipocyte hypertrophy, systemic hypercholesterolemia, inflammation and glucose intolerance [62]. This study supports a beneficial role for LAMs in obesity and potentially in other disease-associated comorbidities such as NAFLD.

\section{Sympathetic Neuron Macrophages-Soaking up Neuronal Insults}

Recently also a subset of sympathetic neuron-associated macrophages (SAMs) within adipose tissue has been discovered [66]. Sympathetic neurons typically produce the neurotransmitter norepinephrine (NE) that facilitates lipolysis and fat mass reduction. Under stress conditions, however, overproduction and systemic NE can lead to hypertension and cardiopathy due to its direct action on cardiovascular tissues [67]. In this regard, Pirzgalska et al., demonstrated that SAMs play a tissue-protective role by scavenging and catabolizing regional NE levels, thereby serving as a local sink that prevents the dangerous effects of systemically increased NE [66]. Such tissue-protective responses have also been described in the intestinal muscularis nerve-associated macrophages that protect against pathogenic insults via B2 adrenergic receptor signaling [68]. In sharp contrast to the anti-inflammatory state of intestinal nerve-associated Cx3cr1-GFP ${ }^{+}$macrophages, SAMs exhibit a pro-inflammatory profile at steady state [66,68]. Regardless of this functional difference, the importance of this population in obesity has been highlighted by the fact that ablation of NE importer, SLC6A2, in these macrophages induced 
weight loss and lipid mobilization [66]. Along the same lines of investigation, a complementary study demonstrated that brown adipose tissue deletion of macrophage methyl-CpG binding protein 2 (MECP2), a factor important in neurodevelopment, resulted in spontaneous obesity [69]. This was attributed to the inhibition of brown adipose tissue sympathetic innervation, and thus a reduction of $\mathrm{NE}$ tissue levels, ultimately leading to altered thermogenesis [69]. Finally, such nerveassociated macrophages appear to be present in a number of different tissues, and at least in mouse models they exhibit a common LYVE $1^{\text {lo }}$ MHCII ${ }^{\text {high }}$ phenotype [70]. This common signature include genes such as $A x l, C c r 2, C x 3 c r 1$ and MHCII-related genes such as H2-DMa, H2-Aa, $H 2-E b 1, H 2-A b 1, C D 74$ and H2-K1 [70]. Combined, these studies highlight the fact that macrophages associated with the sympathetic neuronal system exhibit specialized molecular programs and thus they provide insight into neuronal-macrophage crosstalk mechanisms.

\section{Adipogenic Macrophages-Guiding Adipocyte Formation}

During pathological conditions such as NALFD, where chronic over nutrition prevails, not only the size but also the number of adipocytes increases to compensate for the excessive lipid availability. In this regard, macrophages participate in the (patho-)physiological remodeling of adipose tissue by guiding new adipocyte formation (adipogenesis). Supporting this concept, a recent study implicated an adipogenic- and tissue reorganization role for monocyte-derived Ly6C macrophages that are uniformly distributed throughout the adipose interstitium [59]. Additionally, a population of osteopontin over-expressing adipose tissue macrophages has been demonstrated to establish an adipogenic niche for tissue repair and remodeling in diet-induced obesity models [71]. The authors of this study also demonstrated the importance of this population by showing that osteopontin-deficient mice fail to form these adipogenic nodes. Additional studies are required to further characterize this subset and its importance in obesity and its complications.

\section{Vasculature Macrophages-Gatekeepers to Systemic Circulation}

With the expansion of the adipose tissue, local macrophages have been implicated to direct the formation of new blood vessels and as such, play a role in the process of angiogenesis. These macrophages express lymphatic vessel endothelial receptor 1 (LYVE1) and secrete tissue remodeling factors (e.g., matrix metalloproteinase (MMP)-7, MMP-9, MMP-12), and factors promoting the formation of endothelial cell tubes (e.g., VEGF, PDGF) [72,73]. A recent study provides a comprehensive overview of the transcriptome, phenotype and tissue-localization regarding these vasculature-associated macrophages (VAMs) [63]. More specifically, the authors show that steady-state VAMs display an anti-inflammatory gene signature, are self-maintaining and exhibit an extremely rapid capacity to engulf blood-born macromolecules or noxious catabolites from the 
surrounding adipose tissue [63]. Strikingly, VAMs are suggested to be highly sensitive to cell death since they are rapidly depleted under acute infectious- or metabolic stress [63]. The loss of these valuable macrophages seems to be only transient, as the vascular niche can be repopulated through the recruitment of a monocyte-derived pre-VAM population [63]. Finally, another important study characterized VAMs by reporting that they exhibit a LYVE1 ${ }^{\text {hi }}{ }^{M H C I I}{ }^{\mathrm{lo}}$ phenotype, and that this was a conserved characteristic for infiltrating monocyte-derived cells across a number of different tissues, including fat. Notably, the common gene signature that depicted this population involves genes such as Lyve1, Timd4, CD5l, Fcna and Vsig4 [70]. The authors further demonstrate that LYVE1 ${ }^{\text {hi MHCII }}{ }^{\text {lo }}$ macrophages expressed higher levels of genes involved in blood vessel morphology, leukocyte migration, tissue repair and fibrosis [70]. Arterial LYVE1 macrophages therefore play a role in maintaining normal vasculature structure, an attribute that could be linked to the regulation of collagen production [74]. Importantly, depleting LYVE1 ${ }^{\text {hi MHCII }}{ }^{\text {lo }}$ during the induction of fibrosis, exacerbated inflammation and the degree of fibrosis in the lung and heart of their experimental mouse model [70]. It will be intriguing for future studies to explore whether similar mechanisms are in place during fibrosis and cirrhosis development in chronic liver diseases such as NAFLD.

\section{CONCLUSIONS}

Increasing evidence highlights the close association between adipose tissue macrophage function and their importance in advancing metabolic disorders such as obesity and its complications. Accordingly, adipose tissue macrophage-targeting approaches have underscored their importance in the development of liver inflammation and insulin resistance. Strategies that inhibit the recruitment of macrophages to the adipose tissue compartment are currently being investigated. However, in view of the latest findings regarding the vast diversity of adipose tissuederived macrophage subsets (reviewed here) it becomes clear that these subsets also portray distinct subtissular-associated functions that can be either beneficial or pathological during disease progression. In addition, it remains to be determined whether these subsets represent distinct subsets or represent merely altered functional states of the same cell. It will therefore be crucial for future studies to integrate transcriptional, metabolic and location signatures at a single-cell level in order to clarify these discrepancies and to define highly specific molecular targets. The latter may reveal important mechanisms that could potentially be exploited to counteract the emerging obesity pandemic.

\section{CONFLICTS OF INTEREST}

The authors declare no conflicts of interest. 


\section{FUNDING}

This work was supported by internal funding from the UZ Leuven (KOOR) as well as by the Flanders fund for scientific research (FWO) (G082018N) and research grants from Gilead Sciences. SvdM is a recipient of a clinical mandate from FWO. HK is supported by an international award from Gilead Sciences.

\section{ACKNOWLEDGMENTS}

The authors wish to acknowledge the contribution of Matthew Bird for grammatical proofreading the manuscript.

\section{REFERENCES}

1. Ghaben AL, Scherer PE. Adipogenesis and metabolic health. Nat Rev Mol Cell Biol. 2019;20(4):242-58. doi: 10.1038/s41580-018-0093-Z

2. Saltiel AR, Olefsky JM. Inflammatory mechanisms linking obesity and metabolic disease. J Clin Invest. 2017;127(1):1-4.

3. Korf H, Boesch M, Meelberghs L, van der Merwe S. Macrophages as Key Players during Adipose Tissue-Liver Crosstalk in Nonalcoholic Fatty Liver Disease. Semin Liver Dis. 2019;39(3):291-300. doi: 10.1055/s-0039-1687851

4. Lefere S, Tacke F. Macrophages in obesity and non-alcoholic fatty liver disease: Crosstalk with metabolism. JHEP Rep. 2019;1(1):30-43.

5. Kazankov K, Jørgensen SMD, Thomsen KL, Møller HJ, Vilstrup H, George J, et al. The role of macrophages in nonalcoholic fatty liver disease and nonalcoholic steatohepatitis. Nat Rev Gastroenterol Hepatol. 2019;16(3):145-59. doi: 10.1038/s41575-018-0082-x

6. du Plessis J, van Pelt J, Korf H, Mathieu C, van der Schueren B, Lannoo M, et al. Association of Adipose Tissue Inflammation With Histologic Severity of Nonalcoholic Fatty Liver Disease. Gastroenterology. 2015;149(3):635-48.e14.

7. Nagareddy PR, Kraakman M, Masters SL, Stirzaker RA, Gorman DJ, Grant RW, et al. Adipose tissue macrophages promote myelopoiesis and monocytosis in obesity. Cell Metab. 2014;19(5):821-35.

8. Kosteli A, Sugaru E, Haemmerle G, Martin JF, Lei J, Zechner R, et al. Weight loss and lipolysis promote a dynamic immune response in murine adipose tissue. J Clin Invest. 2010;120(10):3466-79.

9. Bijnen M, Josefs T, Cuijpers I, Maalsen CJ, van de Gaar J, Vroomen M, et al. Adipose tissue macrophages induce hepatic neutrophil recruitment and macrophage accumulation in mice. Gut. 2018;67(7):1317-27.

10. van der Windt DJ, Sud V, Zhang H, Varley PR, Goswami J, Yazdani HO, et al. Neutrophil extracellular traps promote inflammation and development of hepatocellular carcinoma in nonalcoholic steatohepatitis. Hepatology. 2018;68(4):1347-60.

11. Tordjman J, Poitou C, Hugol D, Bouillot JL, Basdevant A, Bedossa P, et al. Association between omental adipose tissue macrophages and liver histopathology in morbid obesity: Influence of glycemic status. J Hepatol. 2009;51(2):354-62. 
12. Solinas G, Vilcu C, Neels JG, Bandyopadhyay GK, Luo J-L, Naugler W, et al. JNK1 in Hematopoietically Derived Cells Contributes to Diet-Induced Inflammation and Insulin Resistance without Affecting Obesity. Cell Metab. 2007;6(5):386-97.

13. Arkan MC, Hevener AL, Greten FR, Maeda S, Li Z-W, Long JM, et al. IKK- $\beta$ links inflammation to obesity-induced insulin resistance. Nat Med. 2005;11(2):191-8.

14. Saberi M, Woods N-B, de Luca C, Schenk S, Lu JC, Bandyopadhyay G, et al. Hematopoietic Cell-Specific Deletion of Toll-like Receptor 4 Ameliorates Hepatic and Adipose Tissue Insulin Resistance in High-Fat-Fed Mice. Cell Metab. 2009;10(5):419-29.

15. Mass E, Ballesteros I, Farlik M, Halbritter F, Gunther P, Crozet L, et al. Specification of tissue-resident macrophages during organogenesis. Science. 2016;353(6304):aaf4238.

16. De Schepper S, Verheijden S, Aguilera-Lizarraga J, Viola MF, Boesmans W, Stakenborg N, et al. Self-Maintaining Gut Macrophages Are Essential for Intestinal Homeostasis. Cell. 2018;175(2):400-15.e13.

17. Hoeksema MA, Glass CK. Nature and nurture of tissue-specific macrophage phenotypes. Atherosclerosis. 2019;281:159-67.

18. Guillot A, Tacke F. Liver Macrophages: Old Dogmas and New Insights. Hepatol Commun. 2019;3(6):730-43.

19. Okabe Y, Medzhitov R. Tissue-Specific Signals Control Reversible Program of Localization and Functional Polarization of Macrophages. Cell. 2014;157(4):832-44.

20. T'Jonck W, Guilliams M, Bonnardel J. Niche signals and transcription factors involved in tissue-resident macrophage development. Cell Immunol. 2018;330:43-53.

21. MacParland SA, Liu JC, Ma X-Z, Innes BT, Bartczak AM, Gage BK, et al. Single cell RNA sequencing of human liver reveals distinct intrahepatic macrophage populations. Nat Commun. 2018;9(1):4383.

22. Van den Bossche J, O’Neill LA, Menon D. Macrophage Immunometabolism: Where Are We (Going)? Trends Immunol. 2017;38(6):395-406.

23. Korf H, du Plessis J, van Pelt J, De Groote S, Cassiman D, Verbeke L, et al. Inhibition of glutamine synthetase in monocytes from patients with acute-onchronic liver failure resuscitates their antibacterial and inflammatory capacity. Gut. 2018;68(10):1872-83. doi: 10.1136/gutjnl-2018-316888

24. Lumeng CN, Bodzin JL, Saltiel AR. Obesity induces a phenotypic switch in adipose tissue macrophage polarization. J Clin Invest. 2007;117(1):175-84.

25. Odegaard JI, Ricardo-Gonzalez RR, Goforth MH, Morel CR, Subramanian V, Mukundan L, et al. Macrophage-specific PPARgamma controls alternative activation and improves insulin resistance. Nature. 2007;447(7148):1116-20.

26. Odegaard JI, Chawla A. Alternative macrophage activation and metabolism. Annu Rev Pathol. 2011;6:275-97.

27. Odegaard JI, Ricardo-Gonzalez RR, Red Eagle A, Vats D, Morel CR, Goforth MH, et al. Alternative M2 Activation of Kupffer Cells by PPAR $\delta$ Ameliorates Obesity-Induced Insulin Resistance. Cell Metab. 2008;7(6):496-507. 
28. Liao X, Sharma N, Kapadia F, Zhou G, Lu Y, Hong H, et al. Krüppel-like factor 4 regulates macrophage polarization. J Clin Invest. 2011;121(7):2736-49.

29. Kelly B, O’Neill LA. Metabolic reprogramming in macrophages and dendritic cells in innate immunity. Cell Res. 2015;25(7):771-84.

30. Van den Bossche J, van der Windt GJW. Fatty Acid Oxidation in Macrophages and T Cells: Time for Reassessment? Cell Metab. 2018;28(4):538-40. doi: 10.1016/j.cmet.2018.09.018

31. Divakaruni AS, Hsieh WY, Minarrieta L, Duong TN, Kim KKO, Desousa BR, et al. Etomoxir Inhibits Macrophage Polarization by Disrupting CoA Homeostasis. Cell Metab. 2018;28(3):490-503.e7.

32. Huang SC-C, Smith AM, Everts B, Colonna M, Pearce ELEJ, Schilling JD, et al. Metabolic Reprogramming Mediated by the mTORC2-IRF4 Signaling Axis Is Essential for Macrophage Alternative Activation. Immunity. 2016;45(4):817-30.

33. Strissel KJ, Stancheva Z, Miyoshi H, Perfield JW, DeFuria J, Jick Z, et al. Adipocyte Death, Adipose Tissue Remodeling, and Obesity Complications. Diabetes. 2007;56(12):2910-8.

34. Lumeng CN, Deyoung SM, Bodzin JL, Saltiel AR. Increased inflammatory properties of adipose tissue macrophages recruited during diet-induced obesity. Diabetes. 2007;56(1):16-23.

35. Han MS, Jung DY, Morel C, Lakhani SA, Kim JK, Flavell RA, et al. JNK Expression by Macrophages Promotes Obesity-Induced Insulin Resistance and Inflammation. Science. 2013;339(6116):218-22.

36. Jha AK, Huang SC-C, Sergushichev A, Lampropoulou V, Ivanova Y, Loginicheva E, et al. Network Integration of Parallel Metabolic and Transcriptional Data Reveals Metabolic Modules that Regulate Macrophage Polarization. Immunity. 2015;42(3):419-30.

37. Tannahill GM, Curtis AM, Adamik J, Palsson-McDermott EM, McGettrick AF, Goel G, et al. Succinate is an inflammatory signal that induces IL-1 $\beta$ through HIF-1a. Nature. 2013;496(7444):238-42.

38. Fang H-Y, Hughes R, Murdoch C, Coffelt SB, Biswas SK, Harris AL, et al. Hypoxia-inducible factors 1 and 2 are important transcriptional effectors in primary macrophages experiencing hypoxia. Blood. 2009;114(4):844-59.

39. Semba H, Takeda N, Isagawa T, Sugiura Y, Honda K, Wake M, et al. HIF-1aPDK1 axis-induced active glycolysis plays an essential role in macrophage migratory capacity. Nat Commun. 2016;7(1):11635.

40. Kim J, Tchernyshyov I, Semenza GL, Dang CV. HIF-1-mediated expression of pyruvate dehydrogenase kinase: A metabolic switch required for cellular adaptation to hypoxia. Cell Metab. 2006;3(3):177-85.

41. Wang $\mathrm{X}$, de Carvalho Ribeiro $\mathrm{M}$, Iracheta-Vellve A, Lowe $\mathrm{P}$, Ambade A, Satishchandran A, et al. Macrophage-Specific Hypoxia-Inducible Factor-1a Contributes to Impaired Autophagic Flux in Nonalcoholic Steatohepatitis. Hepatology. 2019;69(2):545-63.

42. Liu L, Lu Y, Martinez J, Bi Y, Lian G, Wang T, et al. Proinflammatory signal suppresses proliferation and shifts macrophage metabolism from Mycdependent to HIF1 a -dependent. Proc Natl Acad Sci U S A. 2016;113(6): 1564-9. 
43. Palsson-McDermott EM, Curtis AM, Goel G, Lauterbach MAR, Sheedy FJ, Gleeson LE, et al. Pyruvate Kinase M2 Regulates Hif-1a Activity and IL-1 $\beta$ Induction and Is a Critical Determinant of the Warburg Effect in LPSActivated Macrophages. Cell Metab. 2015;21(1):65-80.

44. Reilly SM, Saltiel AR. Adapting to obesity with adipose tissue inflammation. Nat Rev Endocrinol. 2017;13(11):633-43.

45. Gautier EL, Shay T, Miller J, Greter M, Jakubzick C, Ivanov S, et al. Geneexpression profiles and transcriptional regulatory pathways that underlie the identity and diversity of mouse tissue macrophages. Nat Immunol. 2012;13(11):1118-28.

46. Lavin Y, Winter D, Blecher-Gonen R, David E, Keren-Shaul H, Merad M, et al. Tissue-Resident Macrophage Enhancer Landscapes Are Shaped by the Local Microenvironment. Cell. 2014;159(6):1312-26.

47. Gosselin D, Link VM, Romanoski CE, Fonseca GJ, Eichenfield DZ, Spann NJ, et al. Environment Drives Selection and Function of Enhancers Controlling Tissue-Specific Macrophage Identities. Cell. 2014;159(6):1327-40.

48. Serbulea V, Upchurch CM, Schappe MS, Voigt P, DeWeese DE, Desai BN, et al. Macrophage phenotype and bioenergetics are controlled by oxidized phospholipids identified in lean and obese adipose tissue. Proc Natl Acad Sci U S A. 2018;115(27):E6254-63.

49. Xu X, Grijalva A, Skowronski A, van Eijk M, Serlie MJ, Ferrante AW. Obesity Activates a Program of Lysosomal-Dependent Lipid Metabolism in Adipose Tissue Macrophages Independently of Classic Activation. Cell Metab. 2013;18(6):816-30.

50. Kratz M, Coats BR, Hisert KB, Hagan D, Mutskov V, Peris E, et al. Metabolic dysfunction drives a mechanistically distinct proinflammatory phenotype in adipose tissue macrophages. Cell Metab. 2014;20(4):614-25.

51. Coats BR, Schoenfelt KQ, Barbosa-Lorenzi VC, Peris E, Cui C, Hoffman A, et al. Metabolically Activated Adipose Tissue Macrophages Perform Detrimental and Beneficial Functions during Diet-Induced Obesity. Cell Rep. 2017;20(13):3149-61.

52. Cinti S, Mitchell G, Barbatelli G, Murano I, Ceresi E, Faloia E, et al. Adipocyte death defines macrophage localization and function in adipose tissue of obese mice and humans. J Lipid Res. 2005;46(11):2347-55.

53. Haka AS, Barbosa-Lorenzi VC, Lee HJ, Falcone DJ, Hudis CA, Dannenberg AJ, et al. Exocytosis of macrophage lysosomes leads to digestion of apoptotic adipocytes and foam cell formation. J Lipid Res. 2016;57(6):980-92.

54. Haka AS, Grosheva I, Chiang E, Buxbaum AR, Baird BA, Pierini LM, et al. Macrophages Create an Acidic Extracellular Hydrolytic Compartment to Digest Aggregated Lipoproteins. Mol Biol Cell. 2009;20(23):4932-40.

55. Nguyen MTA, Favelyukis S, Nguyen A-K, Reichart D, Scott PA, Jenn A, et al. A Subpopulation of Macrophages Infiltrates Hypertrophic Adipose Tissue and Is Activated by Free Fatty Acids via Toll-like Receptors 2 and 4 and JNKdependent Pathways. J Biol Chem. 2007;282(48):35279-92.

56. Kim K, Shim D, Lee JS, Zaitsev K, Williams JW, Kim KW, et al. Transcriptome analysis reveals nonfoamy rather than foamy plaque macrophages are 
proinflammatory in atherosclerotic murine models. Circ Res. 2018;123(10):1127-42.

57. Spann NJ, Garmire LX, McDonald JG, Myers DS, Milne SB, Shibata N, et al. Regulated accumulation of desmosterol integrates macrophage lipid metabolism and inflammatory responses. Cell. 2012;151(1):138-52.

58. Baardman J, Verberk SGS, Prange KHM, Lutgens E, De Winther MPJ. A Defective Pentose Phosphate Pathway Reduces Inflammatory Macrophage Responses during Hypercholesterolemia. Cell Rep. 2018;25:2044-52.e5.

59. Hill DA, Lim H-W, Kim YH, Ho WY, Foong YH, Nelson VL, et al. Distinct macrophage populations direct inflammatory versus physiological changes in adipose tissue. Proc Natl Acad Sci U S A. 2018;115(22):E5096-105.

60. Li P, Lu M, Nguyen MTA, Bae EJ, Chapman J, Feng D, et al. Functional Heterogeneity of CD11c-positive Adipose Tissue Macrophages in Diet-induced Obese Mice. J Biol Chem. 2010;285(20):15333-45.

61. Ying W, Riopel M, Bandyopadhyay G, Dong Y, Birmingham A, Seo JB, et al. Adipose Tissue Macrophage-Derived Exosomal miRNAs Can Modulate In Vivo and In Vitro Insulin Sensitivity. Cell. 2017;171(2):372-84.e12.

62. Jaitin DA, Adlung L, Thaiss CA, Weiner A, Li B, Descamps H, et al. LipidAssociated Macrophages Control Metabolic Homeostasis in a Trem2Dependent Manner. Cell. 2019;178(3):686-98.e14.

63. Silva HM, Báfica A, Rodrigues-Luiz GF, Chi J, Santos P d'Emery A, Reis BS, et al. Vasculature-associated fat macrophages readily adapt to inflammatory and metabolic challenges. J Exp Med. 2019;216(4):786-806.

64. Keren-Shaul H, Spinrad A, Weiner A, Matcovitch-Natan O, Dvir-Szternfeld R, Ulland TK, et al. A Unique Microglia Type Associated with Restricting Development of Alzheimer's Disease. Cell. 2017;169(7):1276-90.e17.

65. Cochain C, Vafadarnejad E, Arampatzi P, Pelisek J, Winkels H, Ley K, et al. Single-Cell RNA-Seq Reveals the Transcriptional Landscape and Heterogeneity of Aortic Macrophages in Murine Atherosclerosis. Circ Res. 2018;122(12):1661-74.

66. Pirzgalska RM, Seixas E, Seidman JS, Link VM, Sánchez NM, Mahú I, et al. Sympathetic neuron-associated macrophages contribute to obesity by importing and metabolizing norepinephrine. Nat Med. 2017;23(11):1309-18.

67. Schroeder C, Jordan J. Norepinephrine transporter function and human cardiovascular disease. Am J Physiol-Heart Circ Physiol. 2012;303(11):H1273-82.

68. Gabanyi I, Muller PA, Feighery L, Oliveira TY, Costa-Pinto FA, Mucida D. Neuro-immune Interactions Drive Tissue Programming in Intestinal Macrophages. Cell. 2016;164(3):378-91.

69. Wolf Y, Boura-Halfon S, Cortese N, Haimon Z, Sar Shalom H, Kuperman Y, et al. Brown-adipose-tissue macrophages control tissue innervation and homeostatic energy expenditure. Nat Immunol. 2017;18(6):665-74.

70. Chakarov S, Lim HY, Tan L, Lim SY, See P, Lum J, et al. Two distinct interstitial macrophage populations coexist across tissues in specific subtissular niches. Science. 2019;363(6432):eaau0964.

71. Lee Y-H, Petkova AP, Granneman JG. Identification of an adipogenic niche for adipose tissue remodeling and restoration. Cell Metab. 2013;18(3):355-67. 
72. Cho C-H, Jun Koh Y, Han J, Sung H-K, Jong Lee H, Morisada T, et al. Angiogenic Role of LYVE-1-Positive Macrophages in Adipose Tissue. Circ Res. 2007;100(4):e47-57.

73. Pang C, Gao Z, Yin J, Zhang J, Jia W, Ye J. Macrophage infiltration into adipose tissue may promote angiogenesis for adipose tissue remodeling in obesity. Am J Physiol Endocrinol Metab. 2008;295(2):E313-22.

74. Lim HY, Lim SY, Tan CK, Thiam CH, Goh CC, Carbajo D, et al. Hyaluronan Receptor LYVE-1-Expressing Macrophages Maintain Arterial Tone through Hyaluronan-Mediated Regulation of Smooth Muscle Cell Collagen. Immunity. 2018;49(2):326-41.e7.

How to cite this article:

Korf H, Boesch M, Feio-Azevedo R, Smets L, Vandecasteele R, van der Merwe S. Depicting the Landscape of Adipose Tissue-Specific Macrophages and Their Immunometabolic Signatures during Obesity. Immunometabolism. 2020;2(1):e200001. https://doi.org/10.20900/immunometab20200001 